\title{
Extensionality for fusions and pluralities
}

\section{Jeroen Smid ${ }^{1,2}$}

Received: 8 March 2018 / Accepted: 27 October 2018 / Published online: 7 November 2018

(c) The Author(s) 2018

\begin{abstract}
One of the more persistent debates in mereology is whether distinct wholes can have the same parts. Extensional mereologists hold that if there is no part that makes the difference, then there is nothing to distinguish the wholes, so sameness of parts implies identity. Non-extensionalists, however, do think there are cases where distinct wholes share all their parts. This paper argues that the kind of argument non-extensionalists employ can also be levelled against a widely accepted extensionality principle of plural logic. Non-extensionalists thus face a dilemma: either give up the argument for nonextensional mereology, or deny both the extensionality principle of mereology and the analogous principle for plural logic.
\end{abstract}

Keywords Mereology $\cdot$ Extensionality $\cdot$ Identity $\cdot$ Composition $\cdot$ Plural logic $\cdot$ Plural identity

\section{Introduction}

The interrelations between mereological relations and identity give rise to various puzzles, like the Ship of Theseus, Tibbles the cat, and the statue David. Such conundrums reveal that we have conflicting ideas about identity, individuality, and mereological change. On the one hand we want to say that, for example, Tibbles just is her feline tissue. On the other hand, it seems that they are different because Tibbles can survive the loss of her tail while her feline tissue has the tail as an essential part. My focus here is not on particular solutions to such puzzles but rather on the reasoning behind the claim that objects like Tibbles and her feline tissue can have exactly the same proper parts and yet be distinct. I am thus concerned with the debate over extensionality. Non-extensionalists or 'anti-extensionalists' hold that distinct objects can

$凶$ Jeroen Smid

jeroen.smid@gmail.com

1 Department of Philosophy, Faculties of Humanities and Theology, Lund University, Helgonavägen 3, Lund, Sweden

2 Department of Philosophy, School of Social Sciences, University of Manchester, Humanities Bridgeford Street, Manchester, UK 
be mereologically indistinguishable, whereas extensionalists hold that mereological indiscernibility implies identity.

Non-extensionality is inevitable if one accepts each of the following three ideas. The first is that there are at least two kinds of composite object: (real) wholes and (mere) collections. ${ }^{1}$ A real whole is such that it can persist through mereological change, whereas a mere collection has its parts essentially. This distinction is deeply rooted both in common-sense as well as the history of philosophy. (Aristotle seems to make the distinction in Metaphysica Z.17.) The second conviction is that a mere collection and a real whole sometimes have the same spatial parts: a clock, for example, has the same spatial parts as the 'mere fusion' of its parts. The final commitment is that objects do not have temporal parts: the clock we see now is not a temporal part of some temporally and spatially extended entity; it is instead 'wholly present' at each time that it exists. ${ }^{2}$

Extensionalists thus deny at least one of the three claims. A mereological essentialist like Chisholm (1976) denies the first claim and holds instead that all objects have their parts essentially. ${ }^{3}$ Burke (1994) instead denies the second. But most extensionalists do accept the first two claims and instead deny the last one. They thus propose an ontology consisting of four-dimensional objects. In that case extensionality is no problem for they can say that, for example, a clock and the mere fusion of its parts are mereologically distinguishable for they do not have exactly the same temporal parts.

This paper is concerned with the argument commonly provided in favour of the first two convictions. The idea that there are two kinds of composite objects that may have the same proper (spatial) parts is often argued for on the basis of Leibniz's Law.

$$
\begin{aligned}
& a \text { and } b \text { have the same (Premise) } \\
& \text { proper parts } \\
& \text { (II) } \quad \mathrm{Fa} \text { and not- } \mathrm{Fb} \\
& \text { (Premise) }
\end{aligned}
$$$$
\text { (III) } \quad a \neq b
$$

However, as pointed out by various philosophers — most notably Varzi (2000) - the argument is problematic if it is meant to demonstrate that $a$ and $b$ are distinct. The problematic character of the argument is two-fold. First, the intuitiveness of instances of (II) may be due to a de dicto reading of that premise. That is, instances of (II) may seem intuitively plausible due to our linguistic intuitions: we hesitate to say that $b$ is an $F$ due to the terms being used. (Below I will illustrate this in more detail.) In this sense the argument is similar to the following 'argument' for the innocence of Brutus: there was no murderer of Caesar before Caesar was murdered, but Brutus did exist before Caesar was murdered; therefore, Brutus $\neq$ the murderer of Caesar. Hence, the intuitive plausibility of certain instances of (II) may be due to a de dicto reading; but

\footnotetext{
1 These two kinds of object go by different names. The first may also be called 'a compound' or 'a (organic) unity'; the other is sometimes called 'a mere sum', 'an aggregate' or 'a mere fusion'.

2 That these three commitments underpin the non-extensionalist position can be clearly seen in the work of Thomson (1983), whose main argument will inform my discussion.

3 But one might deny the first commitment by holding that there are no such composite objects as 'mere collections' that have their parts essentially. I hint at this strategy at various points in this paper, although it deserves a more systematic treatment at some other occasion.
} 
whether it is appropriate to say of something that it is (not) $F$ has little to do with whether something is (not) $F$. So the de dicto reading does not render the argument valid. Second, the valid-making de re reading (concerning the object(s)) is, however, also problematic, because it begs the question to the person who identifies $a$ with $b$. It begs the question because (II) presupposes that (III) is true, but this latter claim was exactly what was up for debate. So, as long as there is no independent argument for (II) the general argument is either invalid or begs the question (Lewis 1971, 204ff; Jubien 1993, 118ff; Varzi 2000, 291ff).

The debate over extensionality seems to be in a deadlock with the opposing sides accepting different philosophical commitments. Extensionalists are committed to the idea that differences need difference-makers (Goodman 1972, p. 159). In particular, a difference between composites needs a corresponding difference in parts. Nonextensionalists do not necessarily deny the general principle that differences need difference-makers, but they do object to the idea that a distinction between composite objects needs to have a corresponding mereological difference-maker. Instead, there might be a brute difference in kind between (real) wholes and (mere) collections that does not correspond to a difference in the mereological makeup of the objects but only shows up when we take into account other properties of the objects (such as their persistence conditions). I want to try to break the deadlock by comparing the debate over the extensionality principle in mereology with a similar extensionality principle from plural logic that non-extensional mereologist commonly do accept. Using a principle that was dubbed 'the Principle of Uniform Solution' (PUS, yes...) by Priest (1994) and a similar principle against ad hoc responses, I argue that the extensionalist has the upper hand in the debate for she treats similar problems in a more uniform manner.

Some assumptions should be made clear right from the start. First, my aim here is mainly methodological. I wish to show that a general type of argument for the claim that distinct objects can have the same spatial parts should not be employed if a certain extensionality principle in plural logic is accepted. The (desired) conclusion is that extensionality principles should be accepted in both the mereological and the plural logic case. ${ }^{4}$ However, this does not yet tell us which of the three convictions I listed above should be given up. One convert may become a perdurantist, another may deny that there is a real distinction between wholes and mere collections, whereas a third may deny that such objects ever coincide. Such moves are not discussed here. To streamline the discussion, however, I will assume that mereological essentialism is false. This assumption seems fair because the non-extensionalist seems very much committed to the idea that objects can survive mereological change.

Another thesis I will assume to be false is the strong Composition as Identity thesis (CAI) according to which an object is identical with its parts taken together. This is necessary for my discussion because CAI is incompatible with non-extensionalism, hence assuming CAI would beg the question to the non-extensionalist. Moreover, as will become clear, it seems that (non-essentialist) extensional mereology, without CAI, can still give some ontological 'flesh' to the intuitive distinction between a (real)

\footnotetext{
4 The conclusion that extensionality fails in both cases is the one Aaron Cotnoir (personal communication) might want to draw. I hope to convince the reader in Sect. 6.2 that this is heresy.
} 
whole and a (mere) collection. So non-extensionalists who are moved by my argument and join to the extensionalist camp might not have to drop that distinction entirely.

The plan is as follows. In Sect. 2 I introduce extensional mereology and repeat, in some more detail, the standard kind of argument against the extensionality principle(s) of mereology. Section 3 then introduces some notions and principles of plural logic. Section 4 shows that the standard argument against extensional mereology has an obvious counterpart in plural logic. Section 5 explains that one should either deny extensionality both for mereology and plural logic or point out a difference between the two arguments. The reasoning here is backed up by the Principle of Uniform Solution and by a ban on ad hoc responses. Assuming that extensionality should not be denied in plural logic I explore possible relevant differences between the two cases (Sect. 6). Not being able to find any, I conclude that non-extensional mereologists violate the Principle of Uniform Solution and give an ad hoc argument for their position.

\section{Ingredients of general extensional mereology}

General extensional mereology (hereafter: GEM) is the product of Leśniewksi (1916) and, independently, Leonard and Goodman (1940). A somewhat rigorous formulation will be useful for our purposes. Taking ' $<$ ' (for 'is part of') as primitive, we can define various other notions:

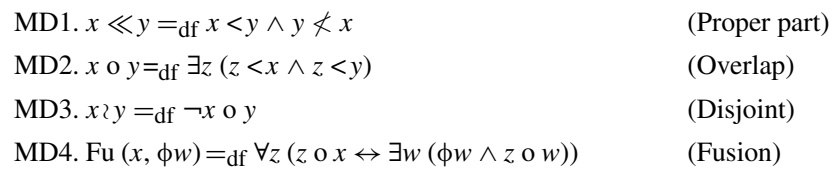

Given these definitions, GEM may be axiomatized as follows:
MA1. $\forall x x<x$
(Reflexivity)
MA2. $\forall x \forall y \forall z((x<y \wedge y<z) \rightarrow x<z)$
(Transitivity)
MA3. $\forall x \forall y((x<y \wedge y<x) \rightarrow x=y)$
(Antisymmetry)
MA4. $\forall x \forall y(y \nless x \rightarrow \exists z(z<y \wedge z z x))$
(Strong Supplementation)
MA5. $\exists w(\phi(w)) \rightarrow \exists x$ Fu $(x, \phi(w))$
(Unrestricted Composition)

Extensionality of Proper Parthood (EP) ${ }^{5}$ follows from these axioms:

MT1. $\forall x \forall y((\exists z, z \ll x \vee \exists z z \ll y) \rightarrow \forall z((z \ll x \leftrightarrow z \ll y) \rightarrow x=y))$

The antecedent of EP is there to ensure that we pick out an object with proper parts. ${ }^{6}$ To see that EP follows from the axioms, note that the contrapositive of Strong

\footnotetext{
5 This formulation of the Extensionality of Proper Parthood can also be found in Simons (1987, p. 112). Sometimes, as in Varzi (2016), the Extensionality of Proper Parthood is formulated with a biconditional as the main connective in the consequent. It then states that composites are identical if and only if they have the same proper parts. I opt here for a conditional instead since the claim that identicals have the same proper parts is not where the controversy lies-especially since this follows from the Indisernibility of Identicals. 6 Without the antecedent the principle would entail that all atoms are identical since they all have the same proper parts.
} 
Supplementation states that if nothing is part of $y$ and disjoint from $x$, then $y$ is part of $x$. If everything that is part of $x$ is also part of $y$, there is nothing that is part of $x$ but disjoint from $y$. So, $x$ is part of $y$. Similarly, if everything that is part of $y$ is also part of $x$, then there is nothing that is part of $y$ but disjoint from $x$. So, $y$ is part of $x$. Hence, by Antisymmetry, $x=y$.

EP is thought to be controversial because objects that have the same proper parts are taken to be distinguishable (in some cases) and hence distinct. As said, the general structure of the argument is as follows:

General argument against EP

$a$ and $b$ have the same proper parts

(III)

$F a$ and not $-F b$

$a \neq b$

As an example, consider the Tinkertoys argument from Thomson (1983). The argument starts with a Tinkertoy house $(\mathrm{H})$ located at the shelf at $1.15 \mathrm{~h}$. Given the existence of the house, the existence of the Tinkertoys, and the principle of unrestricted composition, we have:

(1) H: the Tinkertoy house

(2) W: the fusion of the Tinkertoys

Assuming that $\mathrm{H}$ only has Tinkertoys as parts, EP gives:

(3) $\mathrm{H}=\mathrm{W}$

At 1:30 one of the Tinkertoys (alpha) is removed and replaced by another stick (beta). So at 1:45:

(4) $\mathrm{H}$ is (completely) on the shelf

(5) W is not (completely) on the shelf

Hence, or so the argument goes, $\mathrm{H} \neq \mathrm{W}$ and distinct objects can be mereologically indistinguishable.

To repeat, one common response on behalf of extensionality is that the intuitiveness of (4) and (5) may be merely linguistic (i.e., de dicto). The term 'the fusion of the Tinkertoys' is not suitable to refer to the house at 1.45 and this makes us think that (5) is true. But that does not mean that the house is not identical with that fusion. Compare: 'the murderer of Caesar' does not (seem to) denote Brutus before he killed Caesar but that does not mean that Brutus is not the murderer of Caesar. So (4) and (5) are not obviously true. Worse, assuming (4) and (5) to be true would beg the question to the extensionalist (Varzi 2000; Lewis 1971, 204ff; and Jubien 1993, 118ff). So these premises need to be justified independently.

Thomson seemingly appeals to common-sense as a justification for (4): 'most of us are inclined to think that $\mathrm{H}$ survives replacement of alpha by beta and is still on the shelf at 1:45' $(1983,204)$. For sake of the argument I will accept both that commonsense does provide a justification for (4), and that we should prima facie respect our common-sense judgments. Thomson's reasoning behind (5) seems to be that the fusion of Tinkertoys is a mere sum that has its parts essentially: ' $\mathrm{W}$ is only partly on 
the shelf at 1:45- the fusion of the Tinkertoys on the shelf at 1:15 is partly on the floor at 1:45, since alpha is on the floor at 1:45.' (Ibid.) To repeat: this will not convince the extensionalist who may simply hold that this is due to our linguistic practices: the term 'the fusion of Tinkertoys' may, for example, pick out different entities at different times even though the object that it denotes at 1.15 just is (identical with) the house of Tinkertoys.

Note that a non-extensionalist like Thomson cannot say that it is part of the definition of 'fusion' that W cannot change parts. This reasoning is unavailable because the house (H) also satisfies the definition of 'fusion' while $\mathrm{H}$ can change parts. (Indeed, the reason why non-extensionalist deny extensionalism is because unities and collections are both considered to be fusions but only the first can persist through change of parts.) It seems to me that the only justification that can be given for (5) is again based on common-sense: the fusion of the Tinkertoys is an instance of the kind collection (or 'mere fusion') and this kind of object is seemingly such that it cannot change its parts. So certain common-sense judgments make EP unacceptable to a non-extensionalist like Thomson. ${ }^{7}$

\section{Some logicians only discuss each other}

Forget for a moment individual objects and their parts and focus instead on plural terms that denote multiple individual objects simultaneously. For example, the plural description 'the authors of Principia Mathematica' denotes Russell and Whitehead; whereas the plural name 'the Smiths' denotes the Smith family members. I will say that a plural term can denote a plurality, but by this I mean that it can more than one object simultaneously. Thus in such cases 'plurality' is used as shorthand for more cumbersome expressions but instances of the term 'plurality' can in principle be paraphrased away. For example, the statement 'all pluralities have at least one member' can be rephrased as 'if there are some objects then there is at least one object that is one of them.' So, for example, by saying that 'the Williams sisters' denotes a plurality, I do not mean that it denotes a sort of plural object that is distinct from but consisting of Serena Williams and Venus Williams. Rather I mean that it denotes Serena Williams and Venus Williams together. ${ }^{8}$

Plural logic concerns this way of denoting and may be seen as a natural, plural extension of first-order (singular) predicate logic. Here I won't say anything in its defence. (So a non-extensional mereologist such as Moltmann (1997) who does not

\footnotetext{
7 This leads Thomson (1983, pp. 215-216) to deny Antisymmetry, a move more recently defended by Cotnoir (2010). Another way to avoid EP is by denying both Unrestricted Composition and Strong Supplementation. (Swapping Strong Supplementation for Weak Supplementation is insufficient because Weak Supplementation, Unrestricted Composition, and the partial ordering axioms still entail EP. See Simons (1987, 29ff) and Varzi (2009).)

8 Also, I will say that Serena Williams is a member of the Williams sisters, because Serena is one of the Williams sisters. But this should not be taken as to imply that 'the Williams sisters' denotes an individual set-like object that is numerically distinct from Serena Williams and Venus Williams (taken together). Note, incidentally, that I still do not assume (strong) Composition as Identity! I do not think that 'the Williams sisters' denotes the fusion of which Serena Williams and Venus Williams are part. It simply denotes these two tennis players.
} 
adhere to plural logic may not be moved by the argument of this paper.) Fortunately for me few metaphysicians seem to think that plural logic is in need of some defence in the first place. Indeed, some explicitly accept standard first order (extensional) plural logic while at the same time denying extensionality for mereology. Peter Simons, for example, proposes a kind of plural logic for 'manifolds' (1982, p. 226) or 'multitudes' (2016), but he objects to EP $(1987,112 \mathrm{ff}){ }^{9}$

For my purposes it suffices to explain only a few notions and principles of plural logic. Intuitively, it makes sense to 'start' with singular predicate logic and extend this logic to 'arrive' at a plural logic. So assuming first-order (singular) predicate logic, we introduce a primitive logical predicate ' $\prec$ ' (read 'is one of'), add plural terms (variables ' $x x$ ', ' $y y$ ' and constants ' $a a$ ', ' $b b$ '), use plural quantifiers to bind plural variables, and let predicates take both singular and plural terms as arguments. ${ }^{10}$ The primitive logical predicate ' $\prec$ ' takes a singular term in its first argument-place and a singular or plural term in its second (since a plurality can consist of only one member, the limit of ' $\prec$ ' is equivalent with '='). It can be used to define plural identity:

PD1. $x x=y y={ }_{\mathrm{df}} \forall z(z \prec x x \leftrightarrow z \prec y y) \quad$ (Plural Identity)

A key principle of plural logic is Comprehension:

PA1. $\exists x \phi(x) \rightarrow \exists x x \forall y(y \prec x x \leftrightarrow \phi(y)) \quad$ (Comprehension)

It states that if there is something that satisfies $\phi$, then there is a plurality that contains all and only the objects satisfying $\phi$. Pluralities cannot be empty though:

$\begin{array}{ll}\text { PA2. } \forall x x \exists y(y \prec x x) & \text { (Non-empty) }\end{array}$

A final principle is the following indiscernibility principle:
PA3. $\forall x x \forall y y(\forall z(z \prec x x \leftrightarrow z \prec y y) \rightarrow$
$(\phi(x x) \leftrightarrow \phi(y y)))$
(Indiscernible)

Plural Identity together with Indiscernible gives a plural version of Leibniz's Law:
PT1. $\forall x x \forall y y(x x=y y \rightarrow(\phi(x x) \leftrightarrow \phi(y y)))$
(Leibniz's Law for Plurals)

More importantly for my purposes is that one direction of the definition of plural identity gives the following Extensionality Principle of 'is one of' (EO):

PT2. $\forall x x \forall y y(\forall z(z \prec x x \leftrightarrow z \prec y y) \rightarrow x x=y y)$

\footnotetext{
9 Similarly, it seems, for Thomas J. McKay who develops a plural logic that validates an extensionality principle analogous to the one I will dub 'EO' (2006, chap. 6), but who does not accept EP (because he does not accept Antisymmetry nor Weak Supplementation) for his mereology for stuff (2015, p. 3089).

10 The interpretation of predicates that are satisfied by plural terms is not straightforward. One should, at least, distinguish between a collective and a distributive reading. Fortunately, the distinction will not matter for our discussion.
} 
The similarity with the EP is striking. Interestingly though, I do not know of anyone objecting to EO in print. ${ }^{11}$ The widespread acceptance of EO is interesting because one can raise an argument against EO that is structurally identical to the argument against EP.

\section{Extensionality for everyone}

Consider what I will call 'the Pink Floyd-argument'. In 1967, Pink Floyd consisted of Syd Barrett $(s)$, Nick Mason $(n)$, Richard Wright $\left(r_{1}\right)$, and Roger Waters $\left(r_{2}\right)$. Given the existence of these persons and the comprehension principle we have a plural term for the band members ('BM') and a plural term for the human beings ('HB'):

(6) BM: the band members of Pink Floyd ${ }^{12}$

(7) HB: $s \& n \& r_{1} \& r_{2}$

(Where ' $\&$ ' is a term-connective such that if ' $\alpha$ ' and ' $\beta$ ' are terms, ' $\alpha \& \beta$ ' is a plural term denoting $\alpha$ and $\beta$ together.)

Assuming that only humans can be band members, EO gives:

(8) $\mathrm{BM}=\mathrm{HB}$

In 1969 Syd Barrett leaves Pink Floyd and is replaced by David Gilmour $(d)$. Then on 15 October 1971 the members of Pink Floyd play in the Winterland Ballroom in San Francisco. So on 15 October 1971 we have the following:

(9) BM are (all) in San Francisco

(10) HB are not (all) in San Francisco

Just as it is intuitive that the parts of a Tinkertoy house can change, it is intuitive that the members of a band can change. So (9) seems correct: the members of Pink Floyd are (all) in San Francisco on 15 October 1971. (10) seems equally unobjectionable: Syd Barrett was not in San Francisco at that time, so only some of HB were in San Francisco on 15 October 1971. Thus, or so the argument may go, by Leibniz's law and (9) and (10) we should say that $\mathrm{BM} \neq \mathrm{HB}$. Hence (8) is false, and we thus have a counterexample to EO. To be sure, I don't think this argument raises any real problems for EO-for I think it is a bad argument. But I think the same holds for the argument against EP, which is exactly the point I wish to make. It is wrong to deny EP because of the Tinkertoys argument while at the same time accepting EO despite the Pink Floyd

\footnotetext{
11 As with EP, EO may be formulated as a biconditional instead. Again, the claim that identical pluralities have the same members seems indisputable; hence I opted for a simple conditional instead. (Thanks to an anonymous reviewer for asking me to motivate my choice.).

12 A reviewer for this journal wondered whether 'the band members of Pink Floyd' wouldn't be more naturally interpreted as concerning a group rather than a plurality. There is of course no choice to be made if groups are pluralities. But let's assume that a group is a single thing, whereas a plurality is not. Then it seems to me that 'BM' denotes a plurality and not a group. One reason is that 'BM' when used as a noun phrase in a true sentence seems to refer to some individuals plurally. (For example, 'The band members of Pink Floyd are four in number', and 'The band members of Pink Floyd are not all from the same city'.) Another reason is that plural logicians suggest analogous terms as examples of plural terms: 'the logicians who wrote Principia Mathematica' (Oliver and Smiley 2013, p. 122), and 'the residents of London' (Yi 2006, p. 244). See also Sect. 6.1 below.
} 
argument. This is wrong because the arguments are relevantly similar and, as I will argue in the next section, one should treat similar arguments similarly.

The two arguments are similar in the following respects. First, both use two terms of which it is unclear whether these have the same denotation (i.e. denote the same object/objects). In the Tinkertoys argument these are singular terms: 'the Tinkertoy house' and 'the fusion of Tinkertoys'. In the Pink Floyd argument they are plural: 'Syd Barrett \& Nick Mason \& Richard Wright \& Roger Waters' and 'the band members of Pink Floyd'. Second, both arguments try to show that the two terms have different denotations, because at some point in time these terms denote distinct objects. ${ }^{13}$ Leibniz's Law seems to legitimize, again in both cases, that (the persistence conditions of) the objects/pluralities are different and, hence, that the proposed extensionality principles are flawed.

Third, both arguments beg the question to those who take the two terms to denote the same object/plurality. The extensional mereologist who identifies the Tinkertoy house with the fusion of Tinkertoys, would not accept both (4) and (5). Similarly the plural logician would not accept both (9) and (10). ${ }^{14}$ Related to this is the fact that in both cases the crucial premises enjoy some intuitive plausibility. It is very intuitive to think that a Tinkertoy house can survive some change of parts. However, the term 'the fusion of Tinkertoys' seems to denote an object that cannot survive change of parts. Similarly it is intuitive to think that the members of a band can change, and thus that 'the band members of Pink Floyd' may not always denote the same people. The term 'Syd Barrett \& Nick Mason \& Richard Wright \& Roger Waters' however seems to denote the same four people at all times.

In some more detail: ${ }^{15}$ I don't think the Pink Floyd argument is really a problem for EO, since the plural term 'BM' may be said to denote Syd Barrett, Nick Mason, Richard Wright, and Roger Waters in 1969 while in 1971 it no longer denotes these four people. If we analyse this as 'in 1967: $\mathrm{BM}=\mathrm{HB}$ ' and 'in 1971: $\mathrm{BM} \neq \mathrm{HB}$ ' then, if we want both these claims to come out as true, it seems we have to accept a notion of contingent or occasional identity such that things can be identical at one time, but

\footnotetext{
13 To be sure, there are arguments against EP that do not make use of time: a statue, for example, is said to have aesthetic or modal properties that the piece of matter constituting the statue lacks. Relativizing parthood to times is thus insufficient to convince a non-extensional mereologist to convert to the extensionalist camp. (Thomson (1983), for example, time-relativizes parthood but still opposes to extensionality.) Timerelativizing the is one of relation is equally insufficient to defend EO from all possible counterexamples. For example, Russell is necessarily one of Russell and Whitehead, but Russell does not seem to be necessarily one of the writers of Principia Mathematica. Again, I think such an argument is deeply mistaken. But it seems that the means to dissolve this argument are also applicable to the argument concerning the statue and its matter. However, to streamline the discussion I will ignore modal variants of both the Tinkertoys argument and the Pink Floyd argument. (Thanks to an anonymous reviewer for asking me to clarify this.).

14 A reviewer for the journal pointed out that when we take 'BM' to denote the five men who are or were at some point in time a band member of Pink Floyd, then EO is not violated because in that case 'BM' and 'HB' denote different pluralities. In this respect, too, the argument against EP is similar. If we take 'the fusion the Tinkertoys' to denote the fusion consisting of all Tinkertoys that are or were at some point in time part of the Tinkertoy house then that fusion has both alpha and beta among its parts. The Tinkertoy house, however, never has at one and the same time both alpha and beta as parts. We can then say that the Tinkertoy house $\neq$ the fusion of Tinkertoys without violating EP because these fusions then do not have the same parts.

15 Thanks to an anonymous reviewer for this journal for asking me to clarify this.
} 
fail to be identical at some other time. Instead of going for such a controversial thesis, we could say that since 'BM' is a term that picks out distinct pluralities of objects at different times, while identity is a relation concerning objects, we should distinguish 'BM' as used in 1969 from 'BM' used in 1971. One way we could do this is by indexing the terms: ' $\mathrm{BM}_{1969}$ ' and ' $\mathrm{BM}_{1971}$ '. (There are other, better ways of dealing with terms that change denotation, but that's neither here nor there.) In that case, the statements ' $\mathrm{HB}=\mathrm{BM}_{1969}$ ' and ' $\mathrm{HB} \neq \mathrm{BM}_{1971}$ ' are unproblematic because it's also clear that ' $\mathrm{BM}_{1969} \neq \mathrm{BM}_{1971}$ '. Moreover, if we follow this analysis we don't have a violation of EO nor of Leibniz's Law, because 'BM 1971 are all in San Francisco' is perfectly consistent with 'BM 1969 (=HB) are not all in San Francisco'.

If one gives this response to the Pink Floyd argument, then an extensional mereologist should be allowed to do the same for the Tinkertoys argument. And there too the term 'the fusion of Tinkertoys' may be said to pick out different objects at different times: it picks out the house at $1.30 \mathrm{~h}$, but something different at $1.45 \mathrm{~h}$-but that doesn't show that we have at $1.30 \mathrm{~h}$ two objects (a house and a fusion of Tinkertoys). (This is indeed the common response extensional mereologists have given and which I explained above after giving the Tinkertoys argument.) My point is thus not that EO is a controversial principle of plural logic because of arguments like the Pink Floyd argument. My point is rather that if EP is considered to be a controversial principle of mereology because of an argument like the Tinkertoys argument, then you should also consider EO controversial because of an argument like the Pink Floyd argument. If you respond to that latter argument by saying that it's a bad argument because terms like 'the band members of Pink Floyd' can change denotation, then I am quite willing to accept that - as long as we apply the same analysis to arguments like the Tinkertoys argument. The idea that we should indeed treat similar arguments similarly is the topic of the next section.

\section{SSDD}

The similarity between the two arguments is bad news for non-extensional mereologists who accept EO because, as I will explain here, accepting only one of the two arguments violates two plausible methodological principles. One such principle is Priest's Principle of Uniform Solution. The other is the more general principle that one should avoid ad hoc responses. Given these principles the non-extensional mereologist should either deny EO or point out a relevant difference between the two arguments that justifies treating them differently. In the remainder EO is taken as indisputable. (So, again, a non-extensional mereologist who denies EO has nothing to fear from what follows. ${ }^{16}$ )

Priest suggests the Principle of Uniform Solution when discussing solutions to the paradoxes of self-reference:

\footnotetext{
$\overline{16}$ But a denial of EO poses hard questions: how exactly is plural identity related to 'is one of' if the definition of Plural Identity is false? How can pluralities with the same members be distinct if a plurality just is its members taken collectively?
} 
[I]f two paradoxes are of the same kind, then it is reasonable to expect them to have the same kind of solution. Generalising, it is natural to expect all the paradoxes of a single family to have a single kind of solution. Any solution that can handle only some members of the family is bound to appear somewhat one-eyed, and as not having got to grips with the fundamental issue. (...) [T]o convince ourselves that two paradoxes are of the same kind we must convince ourselves (a) that there is a certain structure that produces contradiction and (b) that this structure is common to the paradoxes. Still, once kindred-ship has been established in this way the point seems undeniable. Let us call it the Principle of Uniform Solution (PUS, sorry): same kind of paradox, same kind of solution. (Priest 1994, p. 32-original italics)

Priest thus advices to solve structurally similar paradoxes in a uniform manner. Note that both the argument against EP and the argument against EO can be stated as paradoxes of extensionality. On the one hand we have an extensionality principle of some kind that motivates identifying the referents of certain terms. But Leibniz's Law together with certain plausible assumptions seems to block this identification. PUS advices us to solve both paradoxes in a similar way. Thus a non-extensional mereologist would violate PUS if she accepts EO but rejects EP because this does not constitute a uniform solution.

Another principle (similar to PUS ${ }^{17}$ ) is the avoidance of ad hocery. Something is ad hoc if it is for one purpose only, which can mean that (i) it is proposed only after being confronted with a problem (i.e. a counterargument, vicious regress, paradox, or objection), (ii) it is an arbitrary exception to a general rule, or (iii) that it lacks independent evidence. ${ }^{18}$ Here the second aspect is what matters, in particular the notion of arbitrariness: accepting an argument $A$ while rejecting a relevantly similar argument $B$ is ad hoc in the sense of being arbitrary. It is arbitrary because there is no reason for treating the arguments differently. Since arbitrary responses are irrational we should avoid them in (philosophical) debates.

Now, if the argument against EP is relevantly similar to the argument against EO, then accepting extensionality in one case but not in the other is a violation of PUS as well as an ad hoc response. The non-extensional mereologist should thus show that there is a (relevant) difference between the arguments that justifies treating them differently. Table 1 presents a summary of the results so far.

\section{No (relevant) difference maker}

My case against the non-extensional mereologist crumbles if there is a relevant difference between the Tinkertoys argument and the Pink Floyd argument. The methodological rules I employ depend crucially on the arguments being relevantly similar. The previous sections showed that there is a strong structural similarity between

\footnotetext{
17 It is possible that it is the rationale behind PUS.

18 For more on being ad hoc, see Wieland (2008). The idea that we should avoid ad hoc ontology drives Rodríguez-Pereyra's (2002) argument for the superiority of resemblance nominalism, Betti's (2015) argument against facts, and my (2017) argument against material constitution.
} 
Table 1 The non-extensional mereologist's predicament

\begin{tabular}{llc}
\hline & Mereological case & Plural logic case \\
\hline Extensionality principle & $\forall x \forall y \forall z(z \ll x \leftrightarrow z \ll y) \rightarrow x=y$ & $\forall x x \forall y y \forall z(z \prec x x \leftrightarrow z \prec y y)$ \\
& $\rightarrow x x=y y$ & \\
Specific instance & $\forall z(z \ll h \leftrightarrow z \ll f) \rightarrow h=f$ & $\forall z(z \prec b m \leftrightarrow z \prec h b) \rightarrow b m=h b$ \\
& $h$ : Tinkertoy-house; $f:$ fusion of & $b m:$ the band members of Pink \\
Tinkertoys & Floyd; $h b: s \& n \& r_{1} \& r_{2}$ \\
Violation of LL & $S h \wedge \neg S f$ & $F b m \wedge \neg F h b$ \\
& $S x: x$ is at the shelf $($ at 1.45 h) & $F x x: x x$ are in San Francisco (in \\
& & $1971)$ \\
Conclusion & Drop EP & $? ? ?$
\end{tabular}

the two arguments. Here I discuss three possible differences between the Pink Floyd argument and the Tinkertoys argument that might justify treating the two arguments differently. ${ }^{19}$

\section{1 'Wrong' ontological intuitions are triggered}

Here is a first possible difference. The Pink Floyd argument is fishy because the terms being used trigger the 'wrong' intuitions whereas this is not the case with the Tinkertoys argument. Note that the claim here cannot be that we mistake our linguistic intuitions about the use of certain terms with ontological intuitions about the object(s) these terms denote. This would be an instance of the de dicto/de re response that is standardly given by the extensionalist when confronted with an argument like the Tinkertoys argument. So in this respect the arguments would be similar, not different. The point is rather that we mistake intuitions about individual objects with intuitions about pluralities of objects because the plural term used in the Pink Floyd argument seems singular. In particular the term 'Pink Floyd', which is part of the complex term 'the band members of Pink Floyd', draws our attention to a band-an individual object. Bands can change parts or members so we mistakenly think that (9) is true because we confuse it with $\left(9^{\prime}\right)$ :

\section{$\left(9^{\prime}\right)$ The band Pink Floyd is in San Francisco}

This last claim is true but concerns an individual (a band) and not a plurality (the members of the band). Since pluralities cannot change members (9) is false even though $\left(9^{\prime}\right)$ is true. There is thus no argument against EO.

The problem is that this does not constitute a difference between the two arguments because we can say something similar about the Tinkertoys argument. This argument is equally fishy because it triggers the wrong ontological intuitions by employing a singular term that seems plural. In particular the term 'Tinkertoys', which is part of the complex term 'the fusion of Tinkertoys', draws our attention to the Tinkertoys-a

\footnotetext{
19 I will not discuss the question begging response that EO is acceptable because all pluralities have their members essentially but that it is not acceptable for individuals because they come in two kinds: unified wholes and mere collections. Theses distinctions are exactly up for debate because the Pink Floyd argument seems to suggest that pluralities also come in two kinds.
} 
plurality. Pluralities cannot change their members so we mistakenly think that (5) is true because we confuse it with $\left(5^{\prime}\right)$ :

$\left(5^{\prime}\right)$ The Tinkertoys are not (all) on the shelf.

This last claim is true but concerns a plurality (the Tinkertoys) and not an individual (the fusion of the Tinkertoys). Since individuals can change parts (5) is false even though $\left(5^{\prime}\right)$ is true. There is thus no argument against EP.

Hence, saying that the Pink Floyd argument is fishy because it triggers the wrong ontological intuitions does not constitute a difference between that argument and the Tinkertoys argument.

\subsection{Pluralities are uncontroversial, fusions are not}

Another possible difference between the two arguments might be that the extensionality principles do not enjoy the same status. Denying EO results in an implausible interpretation of what a plurality is. But EP can be dropped while still having a reasonable notion of what it takes to have proper parts.

To see that EO is simply not up for debate, note that pluralities are meant to be ontologically lightweight objects. Indeed, even though 'a plurality' is a singular term, plural logicians deny that it denotes a single plural object. They think it is a mistake of singularist approaches of plural terms to treat plural terms as denoting a plural object (be it a set or a mereological fusion or something else entirely). We should instead take plural expressions seriously as terms that plurally denote some objects. So, in a very specific (narrow) sense, acceptance of plural logic is an ontologically innocent move because acceptance of plural logic does not change the number (or kind) of elements in one's domain of quantification but only increases one's expressive power by making it possible to plurally denote those elements.

Take, for example, a domain with two elements, $a$ and $b$. What pluralities are taken to be depends crucially on what the term ' $a \& b$ ' is understood to denote. If it denotes a single object that 'consists of' $a$ and $b$, then plural logic does entail a change in the number (and kind) of elements in one's domain. Next to $a$ and $b$ one would then accept a 'plural entity' consisting of $a$ and $b$. This is not the intended interpretation of plural terms in plural logic. What is intended is that ' $a \& b$ ' simply denotes $a$ and $b$ taken together. But this interpretation is unavailable if we were to drop EO. For if it were dropped, pluralities with the same members can be distinct, which means that plural terms cannot just denote some individuals taken together. To see this, note that a domain consisting of $a$ and $b$ can only be a counterexample to EO if $a b \neq b a$, even though $a b$ and $b a$ consist of the same individuals. Thus for ' $a b \neq b a$ ' to be true plural terms should denote something other than just the individuals taken together because the individuals of $a b$ and of $b a$ are the same. So EO is unobjectionable because denying it results in a mistaken conception of a plurality. But, and herein lies a possible difference between the two arguments, EP is not unobjectionable. Denying EP does not seem to result in the wrong conception of a composite object.

But there are at least two problems with using this difference between the two arguments as a justification for treating them differently. First, it is not evident that there is a real difference here, for the extensional mereologist may insist that denying 
EP is only possible given an incorrect conception of a composite object. Indeed, some extensional mereologists sometimes do seem to think that those who deny EP have a different (or incorrect) conception of what it takes to be a composite object. David Lewis, for example, objects to unmereological composition as not being a case of composition at all:

But if what goes on is unmereological, in what sense is the new one composed of the old ones? In what unmereological sense are they present in it? After all, not just any operation that makes new things from old is a form of composition! (...) But if [one] does insist that [t]his unmereological composition is nevertheless composition, in a perfectly literal sense, then I need to be told why. Saying so doesn't make it so. What is the general notion of composition, of which the mereological form is supposed to be only a special case? (Lewis 1999, p. 97)

Bear in mind that what Lewis means by 'unmereological composition' is 'composition not in accordance with the principles of general extensional mereology'. Hence, for Lewis, EP seems to be a necessary aspect of the only proper understanding of a composite object.

Similarly, Achille Varzi thinks that EP is a part of formal ontology:

(...) (EP) is available as a formal, domain independent, metaphysically neutral principle. It is not neutral in the sense that anything goes: this would be of little use. But (EP) is neutral in so far as the link that it establishes between part-whole structures and identity need not depend on any specific views about the nature of what there is, such as the view that material objects are four- or perhaps five-dimensional entities. (Varzi 2008, pp. 122-123)

This does not necessarily mean that Varzi thinks that EP is not up for debate, but it does seem that if it really is a 'domain independent, metaphysically neutral principle' then it could hardly be more controversial than EO.

More generally, extensional mereologists who respond to the Tinkertoys argument and the like seem to think the objection stems from a misunderstanding. In particular, that the non-extensionalist confuses sense (or description) with reference:

Normally we no more conclude that we describe different composite entities when we name two people in different order than we conclude that a house from top to bottom and the house from bottom to top are different entities, or that the capital of Massachusetts and the largest city in New England are different things. (Goodman 1972, p. 164)

Indeed, just as plural logicians may think that denying EO results in a theory that is not really about pluralities, so too an extensional mereologist may hold that denying EP results in a theory that is not really about the part-whole relation.

Second, and more importantly, even if the two extensionality principles are unequal with respect to their disputability, it is not clear how this constitutes a relevant difference between the two arguments. PUS does not seem to license that conclusion, for it focuses on structures. The different levels of (in)disputability, however, do not imply a structural difference between the two arguments. One should of course deny either (9) or (10) in the Pink Floyd argument if EO is not up for debate. But this does not 
change the fact that this argument is structurally similar to the Tinkertoys argument, even if the latter contains a more controversial extensionality principle. Hence, PUS still recommends accepting (or denying) extensionality in both cases.

The same reasoning applies when we focus on our wish to avoid ad hoc responses. If one were to accept extensionality only for pluralities because that extensionality principle is simply not up for debate, then one has to dissolve the Pink Floyd argument in some way. Not doing the same with the Tinkertoys argument still constitutes an ad hoc or arbitrary response. The crucial premises in the Pink Floyd argument-(9) and (10)—enjoy the same (im)plausibility as those in the Tinkertoys argument-(4) and (5) - even if EP is more controversial. The point is that if one accepts intuitiveness as evidence in one case, then one should accept equally intuitive claims in similar cases. Since the Tinkertoys argument and the Pink Floyd argument trigger similar intuitions, it is ad hoc to accept them as evidence only in the case where it contradicts a more controversial principle. Instead, the fact that certain intuitions seem to contradict the indisputable EO should make one wary of similar intuitions that contradict a similar extensionality principle. ${ }^{20}$

So both PUS and our wish to avoid ad hoc responses prefer accepting (or denying) extensionality in both cases, even if one of the two extensionality principles is more controversial than the other.

\subsection{EP can be saved in a way that EO cannot}

It may be that there is a technique to neutralize only one of the two arguments. In particular, it may be that a response from Varzi to block the Tinkertoys argument cannot be used to block the Pink Floyd argument. ${ }^{21}$ It is unclear how much this would help the non-extensional mereologist, because if Varzi's response does indeed vindicate $\mathrm{EP}$, then there is no point in being a non-extensionalist. So it seems that the non-extensionalist should not point out that there is response available to block her argument against EP but that cannot be used to block my Pink Floyd argument against EO. However, it is nonetheless worth considering whether Varzi's response is limited to the Tinkertoys argument (and similar mereological cases) such that it cannot be used to block the Pink Floyd argument (and similar plural logical cases) because this would show that there may be a significant difference between the two arguments.

Let me first explain Varzi's (2008) line of response on behalf of EP. The argument against EP that we used here aims to show that (i) the house is (numerically) distinct from the fusion of Tinkertoys even though (ii) they have the same proper parts. The argument for (i) is based on the idea that once we take away a single Tinkertoy, the house remains at the exact same location, but the mere fusion does not and instead it

\footnotetext{
20 To be sure, I do not think that anyone who takes EO to be uncontroversial should find EP equally uncontroversial. Neither do I wish to hold that the arguments in favour of EO also speak in favour of EP because EO and EP are structurally similar. All I claim is that differences in the level of controversy of $\mathrm{EO}$ and EP or differences between the arguments in favour of EP or EO, are insufficient to hold that only the Tinkertoys argument is sound. It is insufficient because it doesn't change the fact that the Tinkertoys argument is structurally similar to the Pink Floyd argument. (Thanks to an anonymous reviewer for asking me to clarify this.)

21 Thanks to Giorgio Lando for suggesting this line of reasoning.
} 
becomes a scattered object. Even if (i) is correct this only constitutes a counterexample to EP if (ii) holds; i.e. the house and the fusion of Tinkertoys must have exactly the same proper parts. Varzi ingeniously exploits the reasoning for (i) to argue against (ii). ${ }^{22}$ Take for example the roof of the house, which is composed of some Tinkertoys. Let's call the roof's collection of Tinkertoys 'the fusion of roof-Tinkertoys'. It seems that the roof is distinct from the fusion of roof-Tinkertoys for the same reason as that given for the claim that the house is distinct from the fusion of Tinkertoys. Like the house, the roof would also remain at exactly the same location if we were to remove one of its parts. This is because both are real wholes that can survive such a loss. But the fusion of roof-Tinkertoys would not remain at the exact same location for it is a mere collection just as the fusion of Tinkertoys is. Thus the roof is distinct from the fusion of roof-Tinkertoys just as the house is distinct from the fusion of Tinkertoys. The question is whether the roof is a proper part both of the house and a proper part of the fusion of Tinkertoys. Clearly, the roof is a proper part of the house but it seems that it is not a proper part of the fusion of Tinkertoys. ${ }^{23}$ But that means the house and the fusion of Tinkertoys do not have the same proper parts after all: the roof is a proper part of the former, but not of the latter. Thus the Tinkertoy house in relation to the fusion of Tinkertoys does not constitute a counterexample to EP after all.

Varzi's response iterates: the non-extensionalist should distinguish the house from its collection of Tinkertoys; the roof of the house from the roof's collection of Tinkertoys; the dormer of the roof from the dormer's collection of Tinkertoys; etc. In each case we 'discover' a proper part that is only had by one of the two objects-thereby vindicating EP. There is however still a violation of EP possible at the level of objects that have only atomic proper parts. Coincident objects composed of the same two atoms cannot be distinguished by looking for further proper parts because atoms lack proper parts. So the only possible violations of EP are at the almost-atomic level. But this does not help the non-extensionalist much because there might not be any atoms and, even if there are, it is unclear why EP can only ever be violated when objects have only atomic proper parts. (The best way out of this predicament for the nonextensionalist might be to deny Antisymmetry. For a detailed discussion, see Cotnoir (2010).)

If Varzi's response had no obvious counterpart in the plural logic setting, then there would be at least one aspect in which the argument against EP is different from the

\footnotetext{
22 I ignore the question whether the fusion of Tinkertoys is part of the house, but Varzi actually starts with this question and argues that if it is a part of the house, then it is a proper part of $\mathrm{H}$ because of Antisymmetry and the fact that the house is said to be distinct from its fusion of Tinkertoys. However, since the house is not a proper part of itself, the house and its fusion of Tinkertoys do not have the same proper parts. This argument, like the one in the main text depends crucially on Antisymmetry. (See also the next footnote.)

23 One could hold that the roof is part of the collection of Tinkertoys if one follows Thomson (1983) and Cotnoir (2010) in denying Antisymmetry. In that case the house can be part of the collection of Tinkertoys and the collection of Tinkertoys may be part of the house. That is, the house and its fusion of Tinkertoys are mutual parts, even though they are distinct. By Transitivity this means that if the roof is part of house, then the roof is part of the fusion of the Tinkertoys (of the whole house). This would block Varzi's line of response from the get-go.
} 
argument against EO. ${ }^{24}$ In that case the question would be whether it is a relevant difference. However, that question is mute because the structure of Varzi's response can also be used for the Pink Floyd argument. To repeat, the Pink Floyd argument tries to establish a difference between the plurality denoted by 'the band member of Pink Floyd' and the plurality denoted by 'Syd Barrett \& Nick Mason \& Richard Wright \& Roger Waters'. This would provide a counterexample to EO, since everyone that is one of the band members of Pink Floyd is also one of Syd Barrett \& Nick Mason \& Richard Wright \& Roger Waters. Note, first, that there are other pluralities 'inside' the plurality of the members of Pink Floyd. One such plurality is the one denoted by 'the members of the rhythm section of Pink Floyd'. Let's suppose that a rhythm section of a band is just the bassist and the drummer, so in 1967 (as well as in 1971) the plural definite description 'the members of the rhythm section of Pink Floyd' denotes Roger Waters and Nick Mason. Obviously, they are also denoted by the plural term 'Roger Waters \& Nick Mason'.

Moreover, if one thinks that the Pink Floyd argument is convincing, one should also think the following Rhythm-Section-of-Pink-Floyd argument is convincing. This latter argument aims to establish the non-identity between the plurality denoted by 'the rhythm section of Pink Floyd' and the one denoted by 'Roger Waters \& Nick Mason'. The non-identity seems to follow from the following argument. Roger Waters leaves Pink Floyd in 1985 so he does not perform with Pink Floyd at Madison Square Garden in New York on 5 October 1987. This means that Roger Waters \& Nick Mason are not among the people at Madison Square Garden on 5 October 1987-of the two, only Nick Mason is there. But the members of the rhythm section of Pink Floyd (Nick Mason and, at that time, Guy Pratt) are among the people in Madison Square Garden at that time. ${ }^{25}$ So it seems that Roger Waters \& Nick Mason are not identical with the members of the rhythm section of Pink Floyd.

Now for the Varzi-style move. It is obvious that the members of the rhythm section of Pink Floyd are among the members of Pink Floyd. But, it is not evident that the members of the rhythm section of Pink Floyd are among Syd Barrett \& Nick Mason \& Richard Wright \& Roger Waters. This is not obvious because Guy Pratt is one of the members of the rhythm section of Pink Floyd (in 1987), but Pratt is not one of Syd Barrett \& Nick Mason \& Richard Wright \& Roger Waters (at any time). If we follow the Pink Floyd argument, then it turns out that some things that are among the members of Pink Floyd are not among Syd Barrett \& Nick Mason \& Richard Wright \& Roger Waters. For example, as we have just seen, the members of the rhythm section of Pink Floyd are among the member of Pink Floyd (and necessarily so), but the members of the rhythm section of Pink Floyd are not among Syd Barrett \& Nick Mason \& Richard Wright \& Roger Waters. This vindicates EO.

So the Pink Floyd argument against EO can be repeated for pluralities within the original pluralities, thereby vindicating EO. (In exactly the same way as EP was

\footnotetext{
24 As said, this might be a Pyrrhic victory for the non-extensionalist: if the difference between the arguments against EP and against EO is the fact that only the first can be blocked by Varzi's response then this does not provide a good reason to accept the argument against EP but not the argument against EO.

25 Those who hold that Guy Pratt was only playing bass for Pink Floyd but that he was not officially a member of the rhythm section of Pink Floyd should adjust the argument such that Nick Mason is the only person in the rhythm section of Pink Floyd after 1985.
} 
vindicated by a repeated application of a Tinkertoys-style argument.) This means that Varzi's response can be transferred to the context of plural logic so the Tinkertoys argument and the Pink Floyd argument are similar in this respect, too. There is a slight difference though which has to do with the fact that EO concerns a reflexive relation (i.e., is one of is reflexive) whereas EP concerns the irreflexive proper parthood relation. Remember that Varzi's defence of EP breaks down at the level of atoms because at that point there are no further proper parts to 'discover' that establish a mereological difference. But the Varzi-style response to save EO does not break down at any point. To see this, note that plural logic treats the grammatically awkward 'Roger Waters is one of Roger Waters' as always true because 'is one of' is reflexive. But the statement 'Roger Waters is one of the bass player of Pink Floyd' is false at some point in time - and grammatically awkward-because at some points in time Roger Waters is not the bass player of Pink Floyd. Of course this would be a bad argument for the claim that the object denoted by 'the bass player of Pink Floyd' is not identical with Roger Waters. It makes more sense to say that the term 'the bass player of Pink Floyd' does not always denote the same object even if it does pick out Roger Waters in 1967 (and 1971 , for that matter). But that response is exactly the one usually given by extensional mereologist when being confronted with an argument like the Tinkertoys argument. Hence this is simply another aspect of the similarity between the Tinkertoys argument and the Pink Floyd argument.

\section{Conclusion}

In the background of the debate over extensional mereology are two conflicting basic intuitions. One is the idea that there can be no difference without something making that difference. This moves the extensional mereologist to hold that distinct objects cannot have exactly the same proper parts: for if the two objects have the same parts, and stand in the same relation (of being composed of) to those parts, what is it that makes it the case that they are distinct? The other intuition is that some objects are more than just their collection of parts: a Fairphone has a greater unity than just its collection of parts. This motivates the non-extensional mereologist to distinguish, in certain cases, a real whole from a mere collection, even if these objects are composed of the same parts.

My aim here was to further this debate by showing how intuitions very similar to the ones that motivate non-extensional mereologists are generally and, I think, justly neglected in a case very similar to the mereological one. This puts pressure on the non-extensionalist who has to defend why the two similar arguments get a dissimilar treatment. That one should treat similar arguments similarly was motivated by Priest's Principle of Uniform Solutions and our dislike of ad hoc responses. Three possible dissimilarities between the two kinds of argument were discussed, but for each of these I explained that there either was no dissimilarity after all, or not a relevant dissimilarity.

If the non-extensional mereologist does not want to give up the extensionality of plural logic, she should find an argument that is structurally different from the Tinkertoys argument. But this may not be necessary if the non-extensionalist can be convinced that the main intuition underlying her view — that real wholes are different 
from mere collections-is compatible with extensionalism. A common way to reconcile extensionality with the distinction between real wholes and mere collections is to introduce some further parts that are responsible for the distinction, such that real wholes and mere collections never have exactly the same parts. Typically, such further parts are thought to be temporal parts: the Tinkertoys house and the mere fusion of Tinkertoy parts do not have all the same temporal parts and are thus mereologically distinguishable. A second, less-explored route would be to analyse mere collections not as mereological fusions but rather as the pluralities with which plural logic is concerned. According to such an account, real wholes are different from mere collections because a whole is a single object with parts, whereas supposed reference to a mere collection should be paraphrased away using plural reference. For example, 'the collection of Tinkertoys' is then a grammatically singular term, but logically plural since it denotes the Tinkertoys (collectively). So then, strictly speaking, even though there are objects with parts (fusions), there are no 'mere collections' of objects. Instead, when we seem to denote a mere collection of objects we simply plurally denote some objects.

I find this latter approach particularly appealing because it is compatible with mereology and plural logic both being extensional, it is neutral on the existence of temporal parts, and it helps explain the difference between a (real) whole from a mere collection: composition makes the difference! A whole is an individual object that stands in a relation of being composed of to some other objects (its parts). We may refer to those latter objects by way of plural reference. In that case we are referring to those parts, not to some individual object that has those objects as parts. ${ }^{26}$ I believe such an account deserves to be explored in more detail at some other time. Here I only showed that a standard type of argument against the extensionality of mereology could also be raised against the extensionality of plural logic. The conclusion I draw from this is that one should not employ such arguments against extensional mereology. Others might conclude that plural logic should be non-extensional.

Acknowledgements Thanks to audiences at the Mereology and Identity conference (3-5 July 2017, Pisa), the Higher Seminar in Lund, and the Higher Seminar in Linköping. In particular thanks to Massimiliano Carrara and Giorgio Lando for organizing the conference in Pisa, and to Erik Olsson and András Szigeti for their invitations for the seminar in Lund and in Linköping, respectively. Special thanks to two anonymous reviewers for this journal for their valuable comments. Part of my research was generously funded by the Swedish Research Council (Grant Number 2017-06160_3).

Open Access This article is distributed under the terms of the Creative Commons Attribution 4.0 International License (http://creativecommons.org/licenses/by/4.0/), which permits unrestricted use, distribution, and reproduction in any medium, provided you give appropriate credit to the original author(s) and the source, provide a link to the Creative Commons license, and indicate if changes were made.

\footnotetext{
26 Accepting unrestricted composition seems to downplay the significance of composition: every collection stands in the composition relation to some whole (a single whole, according to extensionalists). However, the fact that each plurality is linked by composition to a whole, does not mean that pluralities are no different from wholes. (Only defenders of the strong Composition as Identity-thesis wish to obliterate the distinction between a plurality and a whole.) Moreover, extensional mereology is compatible with restricted composition, so an extensional mereologist may put a restriction of composition if that would make it fit better with her idea of composition being a significant relation.
} 


\section{References}

Betti, A. (2015). Against facts. Cambridge, MA: MIT Press.

Burke, M. B. (1994). Preserving the principle of one object to a place: A novel account of the relations among objects, sorts, sortals and persistence conditions. Philosophy and Phenomenological Research, 54, 591-624.

Chisholm, R. M. (1976). Person and object: A metaphysical study. London: Allen \& Unwin.

Cotnoir, A. J. (2010). Antisymmetry \& non-extensional mereology. Philosophical Quarterly, 60(239), $396-405$.

Goodman, N. (Ed.), (1972). A world of individuals. In Problems and projects (pp. 155-172). Indianapolis: The Bobbs-Merrill Company Inc.

Jubien, M. (1993). Ontology, modality and the fallacy of reference. Cambridge: Cambridge University Press.

Leonard, H. S., \& Goodman, N. (1940). The calculus of individuals and its uses. The Journal of Symbolic Logic, 5(2), 45-55.

Leśniewski, S. (1916). Podstawy Ogólnej Teoryi Mnogosci. I. Moskow: Prace Polskiego Kola Naukowego w Moskwie, Sekcya matematyczno-przyrodnicza.

Lewis, D. (1971). Counterparts of persons and their bodies. Journal of Philosophy, 68(7), 203-211.

Lewis, D. (Ed.), (1999). Against structural universals. In Papers in metaphysics and epistemology (pp. 78-107). Cambridge: Cambridge University Press.

McKay, T. J. (2006). Plural predication. Oxford: Oxford University Press.

McKay, T. J. (2015). Stuff and coincidence. Philosophical Studies, 172(11), 3081-3100.

Moltmann, F. (1997). Parts and wholes in semantics. Oxford: Oxford University Press.

Oliver, A., \& Smiley, T. (2013). Plural logic. Oxford: Oxford University Press.

Priest, G. (1994). The structure of the paradoxes of self-reference. Mind, 103(409), 25-34.

Rodríguez-Pereyra, G. (2002). Resemblance nominalism: A solution to the problem of universals. Oxford: Oxford University Press.

Simons, P. (1982). Plural reference and set theory. In B. Smith (Ed.), Parts and moments—Studies in logic and formal ontology (pp. 199-260). München: Philosophia Verlag.

Simons, P. (1987). Parts: A study in ontology. Oxford: Oxford University Press.

Simons, P. (2016). The ontology and logic of higher-order multitudes. In M. Carrara, A. Arapinis, \& F. Moltmann (Eds.), Unity \& plurality -Logic, philosophy, and linguistics. Oxford: Oxford University Press.

Smid, J. (2017). Material constitution is ad hoc. Erkenntnis, 82(2), 305-325.

Thomson, J. J. (1983). Parthood and identity across time. The Journal of Philosophy, 80(4), 201-220.

Varzi, A. C. (2000). Mereological commitments. Dialectica, 54(4), 283-305.

Varzi, A. C. (2008). The extensionality of parthood and composition. The Philosophical Quarterly, 58(230), 108-133.

Varzi, A. C. (2009). Universalism entails extensionalism. Analysis, 69(4), 599-604.

Varzi, A. C. (2016). Mereology. In E. N. Zalta (Ed.), Stanford encyclopedia of philosophy. https://plato.sta nford.edu/archives/win2016/entries/mereology/.

Wieland, J. W. (2008). Minimize ad hoc turtles. MA Thesis, Amsterdam: University of Amsterdam. http:// dare.uva.nl/cgi/arno/show.cgi?fid=126277. Accessed 1 May 2017.

Yi, B. (2006). The logic and meaning of plurals. Part II. Journal of Philosophical Logic, 35(3), 239-288. 